\title{
Structure, dynamics, and rheology of concentrated dispersions of poly(ethylene glycol)-grafted colloids
}

\author{
Malin Zackrisson, ${ }^{1, *}$ Anna Stradner, ${ }^{2}$ Peter Schurtenberger, ${ }^{2}$ and Johan Bergenholtz ${ }^{1}$ \\ ${ }^{1}$ Department of Chemistry, Göteborg University, SE-412 96, Göteborg, Sweden \\ ${ }^{2}$ Department of Physics, University of Fribourg, CH-1700, Fribourg, Switzerland
}

(Received 12 August 2005; published 24 January 2006)

\begin{abstract}
A comprehensive experimental study of the dynamics and rheology of concentrated aqueous dispersions of poly(ethylene glycol)-grafted colloidal spheres is reported. The study focuses on good solvent conditions, for which excluded-volume interactions dominate. At high concentrations a glass transition is evident from the nondecaying component of the intensity correlation function measured with three-dimensional dynamic light scattering. Results for the linear viscoelastic and steady shear rheology on approaching the glass transition correlate well with the slowing of the diffusive dynamics; in particular, at, or close to, the concentration where the dynamics becomes nonergodic, the dispersions acquire a low-frequency plateau in the elastic shear modulus as well as a yield stress. The overall behavior of the dispersions conforms to that of hard-sphere dispersions; however, some qualitative differences are observed in the evolution of the dynamics and rheology with increasing concentration near the glass transition.
\end{abstract}

DOI: 10.1103/PhysRevE.73.011408

PACS number(s): 82.70.Dd, 83.60.Bc, 83.60.Fg, 64.70.Pf

\section{INTRODUCTION}

The dynamics of concentrated colloidal dispersions is a subject of ongoing research, experimental as well as theoretical. The related topic of dispersion rheology elicits likewise much interest, and it attracts further attention owing to its impact on processing and transport aspects in applications of colloidal systems subjected to flow as well as fundamental interest in out-of-equilibrium colloidal behavior. The rheological response of a dispersion reflects a complicated relationship among microstructure, colloidal-level forces, and applied deformations. While the dilute limiting behavior is well understood [1], there is much yet to be clarified regarding dynamics and rheology at high particle concentrations. Recently seen advances in our understanding of the rheology of solid-particle, particularly hard-sphere, colloidal dispersions have emerged as a result of parallel developments of model colloidal systems [2-4], computer simulation algorithms [5], and theory [6-9].

The connection between equilibrium phase behavior and diffusive dynamics on the one hand and the rheology on the other has been emphasized in past experimental studies [10-12]. In particular, the nature of the tremendous rise in the zero-shear viscosity on approaching, e.g., a critical particle concentration is accompanied by a slowing down of long-time diffusion [13]. Work on hard-sphere colloidal systems [14-17] points to the correlated particle motion associated with the cage effect as the basic mechanism. Two characteristic time scales emerge for sufficiently high concentrations, one connected to in-cage diffusion and a slower one owing to restructuring of cages via intercage particle diffusion. Beyond a glass transition [14-17], while the former persists, the latter is strongly suppressed leading to nonergodic dynamics. Later studies have revealed additional

\footnotetext{
*Electronic address: malinz@chem.gu.se
}

mechanisms at work [18-21], adding to the complexity of the dynamics around the glass transition [22,23], but the system, certainly from a practical viewpoint, behaves as a solid.

The idealized mode coupling theory (MCT) [24-27] incorporates approximations aimed precisely at capturing the cage effect; it makes predictions, essentially free of adjustable parameters, for equilibrium dynamics $[16,17,25-27]$ and linear viscoelastic properties [11,28,29], and models based on extensions of MCT capture a number of nonlinear flow phenomena $[8,9]$. Within MCT, a permanent entrapment of particles ensues at the glass transition, resulting in a transition to an amorphous (nonergodic) solid, characterized by vanishing long-time diffusion, a diverging zero-shear viscosity, a finite, zero-frequency elastic modulus, and a yield stress. Indeed, hard-sphere dispersions appear to acquire a yield stress [30] and a zero-frequency elastic modulus [11], and to exhibit a cessation of large-scale particle motion [21] on crossing the glass transition.

With the hard-sphere system so well characterized, prospects for telling whether more complex systems can be accommodated within the same framework seem promising. Indeed, structural glass transitions are observed in other systems of mutually repulsive colloids, such as charge-stabilized [31] and microgel-particle [32] systems. Thus, at least as far as the equilibrium dynamics goes, there is strong support for colloidal glass formation as a generic process, provided crystallization can be suppressed. Notably, the precise location of the glass transition varies with the nature of the interaction, such that it may set in far away from random close packing of spheres [31].

In this study, we pursue a comprehensive investigation of dispersions of polymer-grafted colloids, encompassing equilibrium statics and dynamics, linear and nonlinear rheology. The aim is to see to what extent the results for this, more complex, colloidal system can be rationalized by the trends now known for hard spheres. This is a commonly chosen approach, using hard spheres as a reference, oftentimes using 
scaling arguments, e.g., to renormalize the volume fraction, to reflect the effect of interactions. Such approaches are often met by a considerable degree of success when a smaller set of properties is under investigation, at least when the interaction is not too soft [33]. The basic question is whether this is an appropriate method when examining a greater range of properties.

Polymer grafting is a common strategy for stabilizing colloids $[1,34]$. Work has been done on the short-time dynamics [35], high-frequency [36] and high-shear [37,38] rheology of polymerically stabilized colloids; additional studies of the low-frequency, linear viscoelastic [39-43] and low-tointermediate shear rheology $[40,41,43-48]$, are of particular relevance to the work presented here. The long-time dynamics, however, has not been investigated in any great detail and never in conjunction with rheological and static structure measurements.

In this work we report on dynamical and rheological measurements of a well characterized aqueous system of poly(ethylene glycol)- (PEG-) grafted polystyrene colloids. Interactions are modulated by solvent quality and range from repulsive under good solvent conditions to attraction dominated under marginal or poor solvent conditions. Solvent quality is regulated by either temperature or addition of salt, usually a combination of the two. In this work we limit the study to good solvent conditions, for which excluded-volume interactions dominate, because the phase diagram is then very simple. The effects of switching to marginal solvent conditions will be reported elsewhere.

In what follows, we begin by giving an account of the dispersions used and the experiments conducted. We proceed by briefly discussing some small-angle neutron scattering measurements of the static structure, followed by the results of dynamic light scattering. Application of steady and smallamplitude oscillatory shear probes the interplay between structural relaxation and time scales associated with the deformation. Just as for noncrystallizing dispersions of hard spheres, a glass transition is found to govern the overall behavior.

\section{EXPERIMENT}

\section{A. Materials}

The colloidal system investigated here consists of poly(ethylene glycol)-grafted, polystyrene nanospheres dispersed in water. We have added $10 \mathrm{mM}$ background electrolyte $\left(6.15 \mathrm{mM} \mathrm{NaCl}+3.85 \mathrm{mM} \mathrm{NaN}_{3}\right)$ to make sure that any surface charges are screened and to prevent bacterial growth during longer-time storage. Further details of the synthesis, cleaning, and characterization have been described previously [49] and will only be summarized briefly here. The particles were synthesized in a one-step copolymerization between styrene and methyl poly(ethylene glycol) acrylate using potassium persulfate as initiator, following the procedure by Brindley et al. [50]. This protocol produces sterically stabilized, spherical latex particles with a core-shell structure. Two batches were synthesized and prepared in an identical manner, one batch used in rheological measurements and the other in dynamic light scattering (DLS) experiments.
Concentrated dispersions were obtained from dilute dispersions by centrifugal filtration (Pall, Gelman Lab.) to sufficiently high mass content. The final weight fractions of stock solutions, determined by drying in an oven at $60{ }^{\circ} \mathrm{C}$, were converted to particle mass concentrations $c(\mathrm{~g} / \mathrm{ml})$ using measured particle densities.

\section{B. Three-dimensional dynamic light scattering}

DLS measurements were performed using the 3D crosscorrelation technique [51,52], on a 3D single-mode fiber goniometer system (LS Instruments) equipped with a Flex correlator and a diode laser with a wavelength of $680.4 \mathrm{~nm}$. The temperature was regulated by a circulating water bath at $25.0^{\circ} \mathrm{C}$ and a scattering angle $\theta$ of $90^{\circ}$ was maintained in all measurements shown here. However, angular scans, covering $30^{\circ}<\theta<130^{\circ}$, were performed to confirm the $q$ independence of the apparent diffusion coefficient. Here, $q$ $=(4 \pi n / \lambda) \sin \theta / 2$ is the magnitude of the wave vector, given in terms of the solvent refractive index $n$ and the laser-light wavelength in vacuo $\lambda$. To suppress multiple scattering further, we used high-quality NMR tubes with a diameter of $5 \mathrm{~mm}$ as sample cells (Armar AG, Switzerland). Ensemble averaging of the signal from nonergodic and very slowly relaxing samples was achieved, following the procedure devised by Xue et al. [53], by continuous rotation of the sample cuvettes. In addition, the use of highly symmetrical sample tubes ensured symmetrical rotation.

\section{Rheology}

Rheological measurements were carried out on a temperature-controlled rotational rheometer (Paar Physica MCR 300 Universal Rheometer) using a cone-plate geometry (50 $\mathrm{mm}$ cone diameter, $1^{\circ}$ cone angle) in controlled strain mode. The rheometer was equipped with direct strain oscillation [54], which adjusts the strain value directly on the sine wave. The samples were protected against drying by a standard solvent trap, with a lid clad on the inside with moistened cloth. Steady-state conditions and reproducibility required long waiting times (up to $12 \mathrm{~h}$ ) for the highest concentrations. The approach to stable measuring conditions was monitored by low-strain, constant frequency dynamic oscillation. After having obtained time-independent moduli, amplitude and frequency sweeps were recorded, on both advancing and receding values of the strain and frequency. The samples were found to be sensitive toward exposure to air, most likely due to oxidative attack [55]. On filling the solvent trap with inert argon gas to limit air exposure, stable measuring conditions were obtained. Similar observations have been made for aqueous solutions of ethylene oxidebased surfactants $[56,57]$. Provided the atmosphere inside the solvent trap was filled with argon gas and the sample was carefully loaded using a microliter glass syringe (Hamilton) for precise volume control, steady-state conditions were reached when enough time for samples to recover from loading was given. The following protocol delivered stable measurements and reproducible data: (i) samples were allowed to recover from loading; (ii) after time testing and no observable drift in the moduli over several hours, amplitude sweeps 
were performed to identify the linear response regime; (iii) fixed-amplitude frequency sweeps in the linear regime were conducted; and (iv) rotational, steady-shear measurements were performed as a last measurement, ensuring that the linear viscoelastic moduli were not recorded after processing by large-deformation, steady shear.

\section{Small-angle neutron scattering}

Small-angle neutron scattering (SANS) experiments were carried out at the large-scale structure diffractometer D22 at the Institut Laue Langevin (Grenoble, France). The details of the SANS measurements and the analysis have been reported elsewhere [49], and we refer to this study for details on the samples and experiments.

\section{RESULTS AND DISCUSSION}

We conduct a study of aqueous dispersions of PEGgrafted spheres of polystyrene. The emphasis will be on the long-time, low-frequency dynamics of concentrated systems. To this end we make use of small particles, such that the Brownian relaxation time in dilute systems, $a^{2} / D_{0}$, in terms of the radius $a$ and Stokes-Einstein diffusion coefficient $D_{0}$, is fast. In addition, sedimentation is then of little concern. The study is limited to low ionic strengths, just sufficient to screen residual surface charges, and room temperature; the solvent quality is then excellent for the PEG graft, such that excluded-volume interactions dominate. Indeed, the shorttime, collective diffusion coefficient increases as a function of particle concentration in the dilute limit, as expected for repulsive interactions [58].

\section{A. Static structure and dynamics}

In earlier work we have characterized the system in some detail using small-angle neutron scattering (SANS), from which some results are shown in Fig. 1. Contrast variation reveals that the polystyrene core radius is $a \approx 21 \mathrm{~nm}$, with a PEG layer thickness of $\delta \approx 4 \mathrm{~nm}$. The particles are moderately polydisperse, $\approx 13.5 \%$. Similar values are to be expected for the particles in this study, as they have been synthesized and prepared under the same conditions. In Fig. 1 the effect of particle concentration on the scattered intensity is shown. Concentration is reported in terms of the mass concentration of particles $c$. As seen, the scattered intensity at low concentration is entirely due to the form factor (intraparticle scattering), but on increasing particle concentration structure factor effects rapidly become pronounced. The suppression of scattering at low $q$ and the emergence of a peak at intermediate $q$ are characteristic of the buildup of positional correlations owing to excluded-volume interactions. Indeed, the structural evolution can be reasonably well captured by an effective hard-sphere model based on PercusYevick theory, provided that the interaction diameter $\sigma^{H S}$ is permitted to assume smaller values than $2(a+\delta)$ for concentrations above $c=0.21 \quad\left(\phi^{H S}=0.34\right)$, suggesting some layer compression and a softer interaction. At the highest concentration in Fig. 1, corresponding to $\phi^{H S}=0.56$, this amounts to roughly a $1 \mathrm{~nm}$ compression of PEG layers. More specifi-

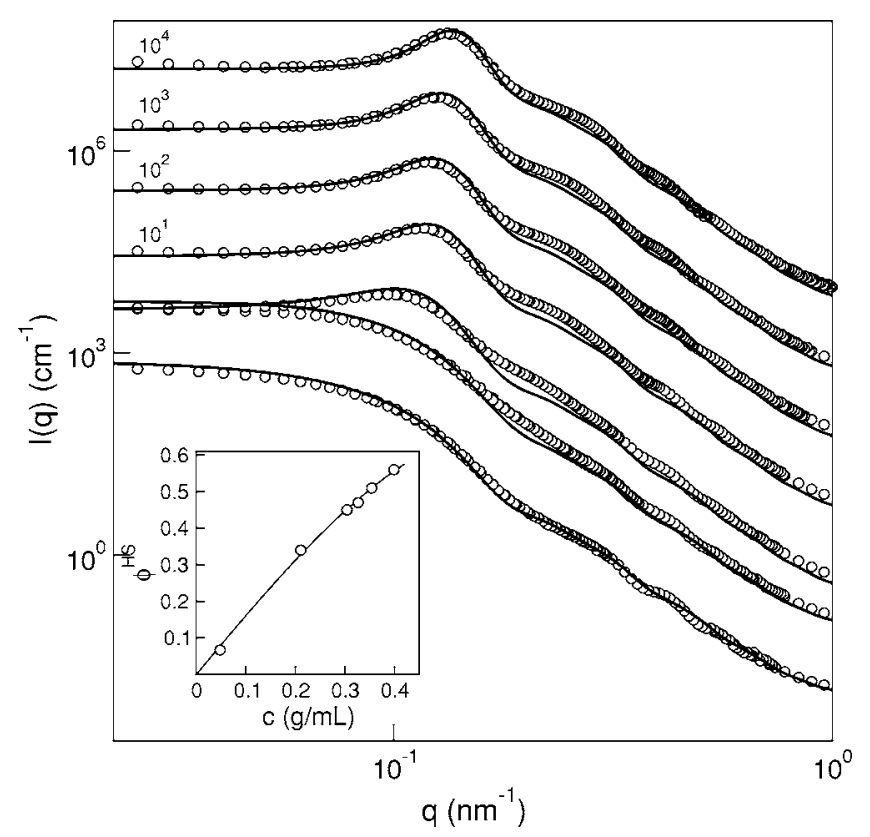

FIG. 1. Scattered intensity as a function of $q$ and mass concentration, from top to bottom, $c=0.40,0.35,0.33,0.30,0.21,0.048$, and $0.0042 \mathrm{~g} / \mathrm{ml}$, corresponding to $\phi^{H S}=0.56,0.51,0.47,0.45$, $0.34,0.067$, and 0.0069 , where data have been taken from [49]. The highest concentrations have been shifted for clarity, with the shift factors given along the left-hand side. Lines are fits to the data, using a core-shell-like contrast profile for the form factor together with an effective hard-sphere model based on the Percus-Yevick solution for polydisperse hard spheres. The inset shows the effective hard-sphere volume fraction $\phi^{H S}$ as a function of mass concentration $c$. The solid line is a fit to a cubic polynomial resulting in $\phi^{H S}=1.632 c-0.086 c^{2}-1.281 c^{3}$.

cally, we make use of the analytical Percus-Yevick solution for hard-sphere mixtures first obtained by Lebowitz [59], as formulated by Blum and Stell [60,61], and size polydispersity is handled using the method of D'Aguanno and Klein [62].

As the particles are small, the absence of crystallization could not be assumed a priori, as visual detection of crystallites would have been impossible. But the degree of polydispersity obtained from the SANS analysis ensures that crystallization does not occur, as is also evident from the broad primary peak in the concentrated samples in Fig. 1; identical scattering results were also obtained $14 \mathrm{~h}$ later. In the inset we report the effective hard-sphere volume fractions $\phi^{H S}$ $=\pi n\left\langle\left(\sigma^{H S}\right)^{3}\right\rangle / 6$ obtained from the data modeling, as a function of the mass concentration. The $\phi^{H S}$ values far exceed those that one obtains from measurement of the particle density [49]; they show a nonlinear dependence on $c$, such that increasing $c$ at high concentration does not result in as much of an increase in $\phi^{H S}$. The same qualitative trend has been noted in the past for polymer-grafted systems [1].

Turning to investigations of the dynamics, we emphasize that although the particle size is small, the high refractive index difference between particles and solvent leads to multiple scattering. We make use of the 3D cross-correlation technique $[51,52]$ to detect singly scattered light. The intensity correlation function $g^{(2)}(\tau)$ is shown in Fig. 2 as a func- 


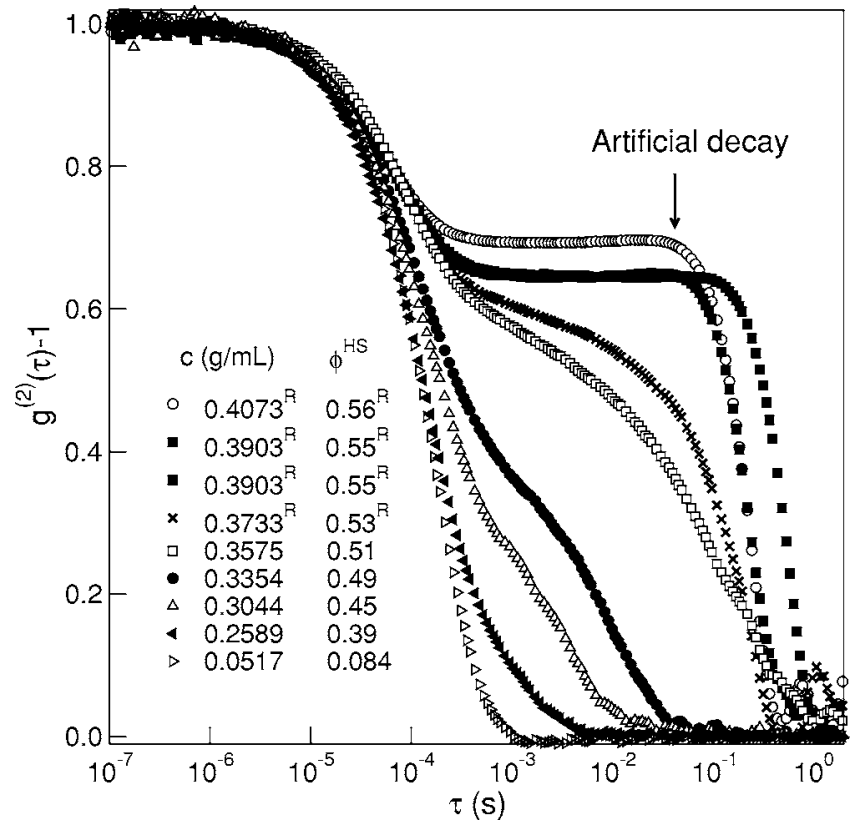

FIG. 2. 3D cross-correlation functions shown as functions of time and concentration, as labeled. As described in the text, and as marked by a superscripted $R$ in the legend, the signals from the three highest concentrations $c=0.4073,0.3903$, and $0.3733 \mathrm{~g} / \mathrm{ml}$ were partially or completely nonergodic and were rotated at $0.05 \mathrm{rpm}$. For $c=0.3903 \mathrm{~g} / \mathrm{ml}$, data recorded for rotation at $0.02 \mathrm{rpm}$ are also included. The additional (artificial) cutoff decays from sample rotation appear at $\tau_{\text {cutoff }} \approx 0.1 \mathrm{~s}$ for $0.05 \mathrm{rpm}$ and $0.2 \mathrm{~s}$ for $0.02 \mathrm{rpm}$.

tion of time and particle concentration. To begin with it is worth noting that the intercept $g^{(2)}(\tau=0)-1$ reduces to less than its theoretical value of 0.25 (for the $3 \mathrm{D}$ setup) reflecting the amount of multiple scattering. The data shown in Fig. 2 have been normalized with the measured intercepts at each concentration. The intercept varies inversely with the turbidity and shows a minimum at intermediate concentrations, qualitatively consistent with the data in Fig. 1. At the highest concentration in Fig. 2, the sample is nearly transparent.

The dynamic measurements were recorded for $q$ $=0.016 \mathrm{~nm}^{-1}$, which, owing to the small particle size $(q a$ $\approx 0.33$ ), is well below the value where the primary structure factor peak is positioned, $q \approx 0.12 \mathrm{~nm}^{-1}$; in fact, the data in Fig. 2 are recorded for a wave vector smaller than the smallest wave vector reached in the SANS measurements. It follows that we measure temporal correlations of large spatial fluctuations in concentration, far larger than those corresponding to the cage size. Measurements at several scattering angles reveal that the apparent time-dependent diffusion coefficient is independent of scattering angle, suggesting that our measurements are effectively done in the $q \rightarrow 0$ limit. As such, one would expect to measure the collective diffusion coefficient. As seen more clearly in Fig. 3, it is possible to define two diffusion constants from our data, one characteristic of the initial decay and a second connected to the final decay into the noise level. It is now known that such a distinction between short- and long-time collective diffusion coefficients exists, though up to $\phi^{H S}=0.4$ the difference be-

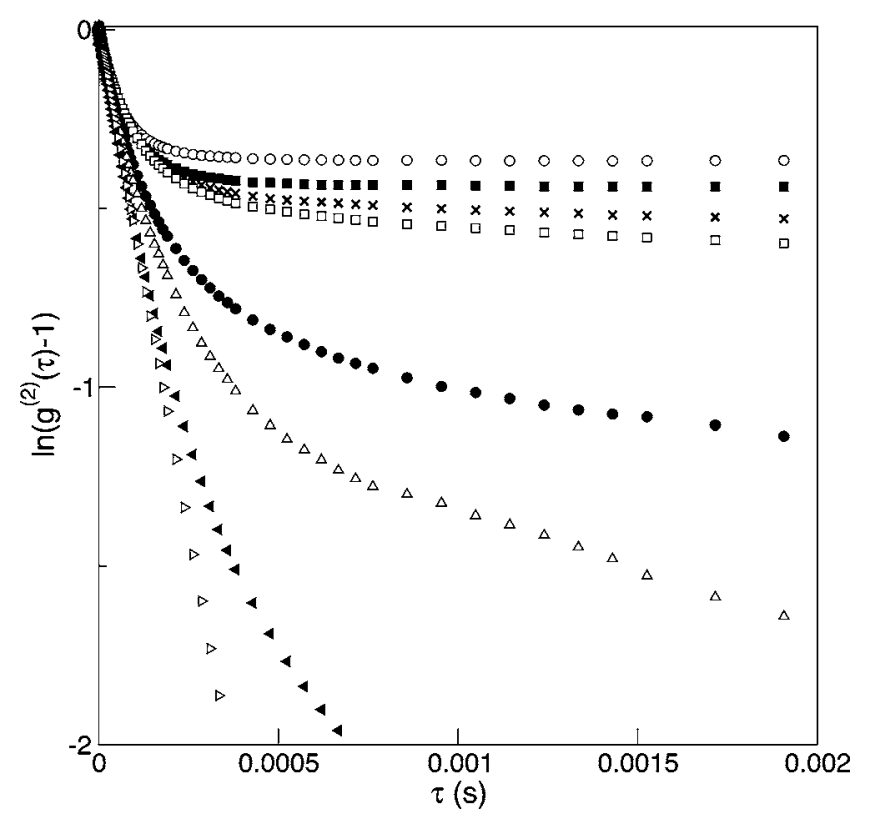

FIG. 3. Semilogarithmic representation of the cross-correlation functions from Fig. 2 as functions of time, showing the same data and with symbols as in Fig. 2.

tween the two is small [63]. However, it is also known that even small degrees of polydispersity tend to introduce selfdiffusion relaxation modes [64], particularly pronounced when $S(q) \ll 1$ for the sampled wave vector, as occurs here on increasing particle concentration. Therefore, at the high concentrations of interest, we are not measuring a true longtime, collective diffusion coefficient, but that is not a concern in the qualitative discussion we pursue in this work.

At low particle concentrations the particle positions decorrelate within a short time span, leading to nearly exponential decay of the intensity correlation function. As concentration is increased a second slower relaxation emerges, which slows down as the concentration is increased further. For the two highest concentrations in Fig. 2, we cannot detect any decay but the initial, fast mode. Consequently, these samples exhibit nonergodic dynamics, at least over the experimental time window, and they require proper ensemble averaging. The three highest concentration samples $\phi^{H S}=0.56,0.55$, and 0.53 were ensemble averaged by slowly rotating the sample cuvettes [53] at $0.05 \mathrm{rpm}$, which also introduces an artificial decay setting in at $\approx 0.1 \mathrm{~s}$. This decay identifies the baseline of the measurement and establishes the height of the plateau, sometimes referred to as the nonergodicity parameter or glass form factor [25]. The plateau value did not vary with rotational speed (cf. sample $\phi^{H S}=0.55$ in Fig. 2).

The measured static structures bracket the concentration span over which the dynamics data in Fig. 2 go from being ergodic to nonergodic. In light of the disordered nature of the static structure, the DLS data exhibit all the characteristics of a glass transition, qualitatively the same as for hard-sphere [14-17], charged-sphere [31], and microgel [32] colloids. Comparing with the static structures in Fig. 1, we locate the nonergodicity transition at around $0.53<\phi^{H S}<0.55(0.373$ $<c<0.39$ ). We attach no special significance to this value being somewhat lower than the experimental hard-sphere 
transition value $\phi^{H S} \approx 0.58[17,21]$, in that we do not expect hard-sphere behavior and in that the data in Fig. 2 differ from those of hard spheres in more ways than one. First, the plateau value is more than a factor of 2 higher than what can be expected (based on MCT) for monodisperse hard spheres at small $q$; this is likely due to polydispersity and incoherent scattering effects [64]. Also, the intensity correlation function evolves with concentration in a somewhat different manner. The slowly decaying mode not only slows with increasing concentration, its amplitude grows rather strongly with concentration as well, at least to a greater extent than what is observed, albeit at larger $q a$, for hard spheres [17]. The appearance of a slow mode correlates well with the failing of a strict hard-sphere modeling of the SANS data starting at $0.34<\phi^{H S}<0.45$, beyond which we must allow for a smaller interaction diameter, $\sigma^{H S}$, than $2(a+\delta)$. Presumably, in this concentration span the softness of the polymer-graft becomes important.

\section{B. Steady shear viscosity}

Having detected how these dispersions undergo a glass transition, yielding a non-relaxing intensity correlation function with 3D DLS, the nonergodic dynamics should also be manifest in the flow behavior. On approaching a glass transition new time scales intrinsic to the system emerge, and one can expect that the rheology is now determined to a large extent by the interplay between these and the time scale over which the structure is perturbed away from equilibrium. For concentrated colloidal dispersions subjected to steady shear we expect that the extent of the low-shear, Newtonian plateau is progressively restricted to lower shear rates as the concentration increases, e.g., $[12,47,65,66]$. Flow curves as functions of particle concentration are shown in Fig. 4. At low concentrations we indeed observe a plateau viscosity at low shear rates, before shear thinning ensues at higher shear rates. Owing to the small particle size, Brownian motion is very effective in keeping the system close to equilibrium, necessitating the application of large shear rates before shear thinning sets in at the lower concentrations. Note, however, that in terms of $\phi^{H S}$ these concentrations would normally be considered rather high.

The situation changes above $\phi^{H S}=0.48$, above which the steady-shear rheology no longer evolves in a textbook fashion. Recall that for such concentrations there is now a clear two-step decay in the intensity correlation function. The lowshear Newtonian plateau disappears, perhaps by being shifted to lower shear rates than those accessible experimentally, but it appears as if a second shear thinning regime at low shear rates emerges instead. This is in contrast to what is generally observed for concentrated dispersions, which usually exhibit a continuous shifting of the Newtonian plateau to higher viscosity values and lower shear rates; see, e.g., $[12,47,65,66]$. In the double-logarithmic plot, it is clear that the shear thinning viscosity only obtains a $\dot{\gamma}^{-1}$ dependence at yet higher concentrations, at around $\phi^{H S} \approx 0.55$, where the viscosity is $10^{7}-10^{9}$ times the solvent viscosity at the lowest shear rates. This concentration is somewhat above, but very close to, the concentration at which the dynamics change to

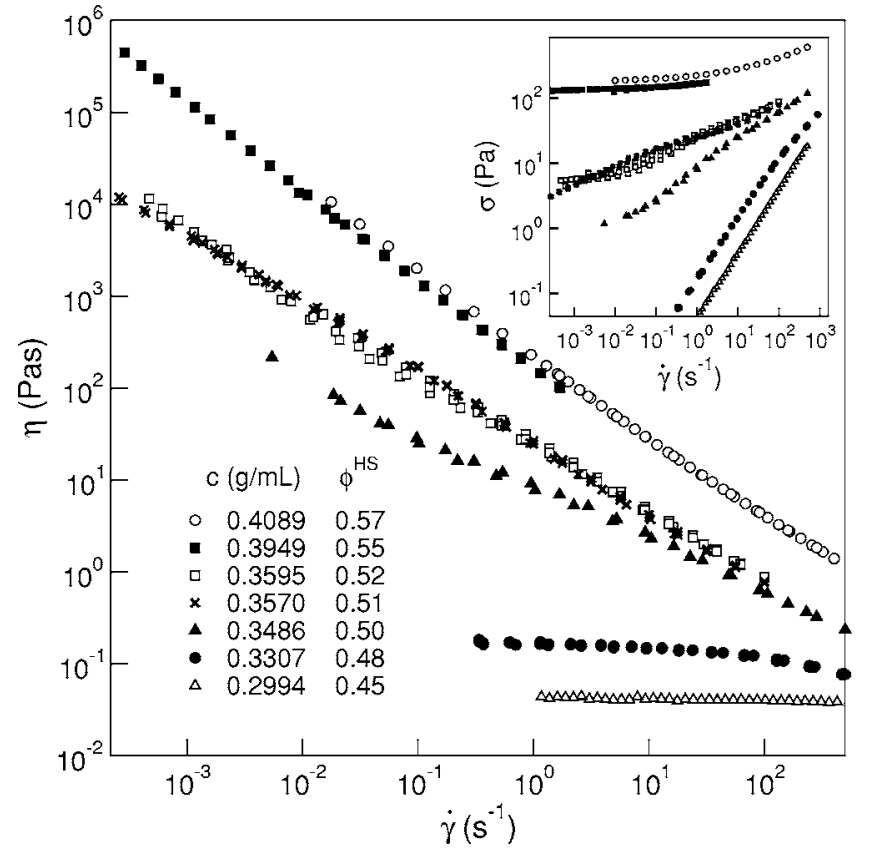

FIG. 4. Viscosity shown as a function of shear rate and particle concentration, as labeled. The inset shows the same data in terms of the shear stress as a function of shear rate.

being nonergodic in Fig. 2. Thus we observe differences with both hard-sphere behavior and other polymerically stabilized particle systems [47], but the changes appear to correlate well with the dynamics. A somewhat similar disappearance of the low-shear plateau with increasing concentration has been reported by Ploehn and Goodwin [40] (for concentrations below the equilibrium crystallization boundary) for dispersions of PEG-grafted colloids. Also, Nommensen et al. [43] observe an abrupt disappearance of the low-shear Newtonian plateau for dispersions of particles with longer grafted polymer layers and Frith et al. [67] note distortions in the shear thinning regime similar to but more pronounced than those we observe. Above the nonergodicity transition, the scaling of the viscosity with shear rate at low shear rates suggests the onset of a yield stress, with a value of $\sigma_{y}$ $\approx 200 \mathrm{~Pa}$. We note that the appearance of such a yield stress for glassy systems is predicted on the basis of an extended, idealized MCT approach to nonlinear rheology $[8,9]$.

\section{Linear viscoelastic behavior}

The influence of the dynamics around the glass transition on the response to a small-amplitude, oscillatory shear has been studied. To identify the linear viscoelastic response regime, strain amplitude sweeps at constant frequency were measured, the results of which are shown in Fig. 5 in terms of the (elastic shear) storage and (viscous) loss moduli, $G^{\prime}$ and $G^{\prime \prime}$. While the response is linear, with $G^{\prime}$ and $G^{\prime \prime}$ assuming constant values, up to very high strains at concentrations below $\phi^{H S} \approx 0.48$, samples at higher concentrations are fluidized beyond a certain strain value, $\gamma_{c}$, such that the elastic modulus decreases and the viscous modulus increases. This behavior is typically observed for concentrated colloidal dis- 


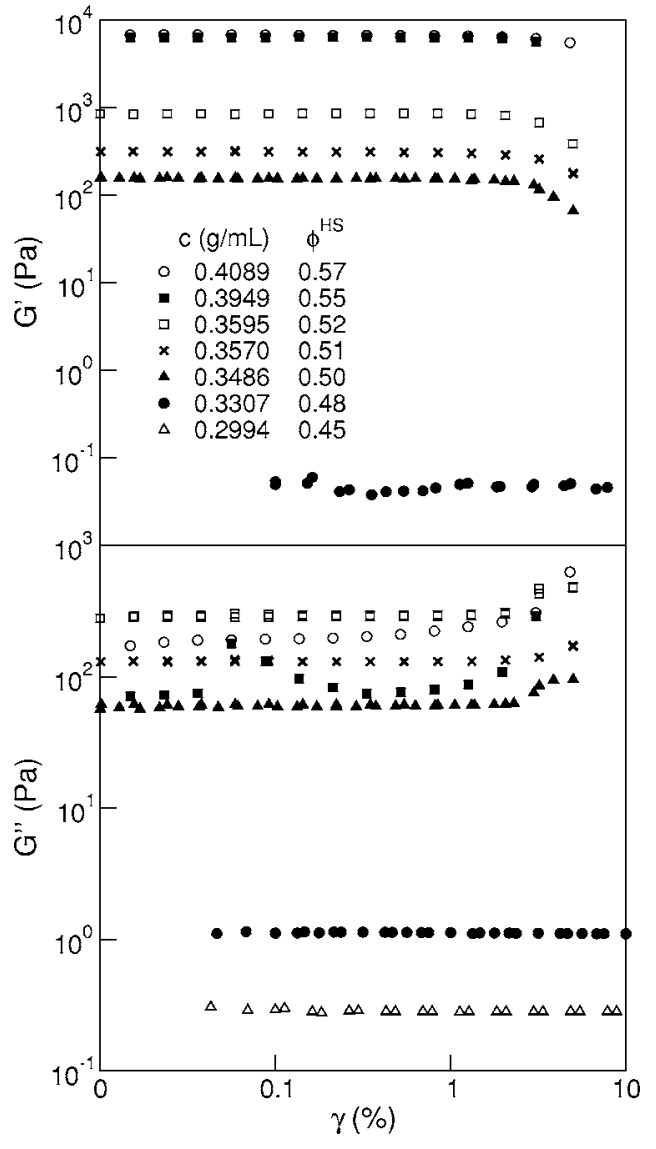

FIG. 5. The linear viscoelastic moduli $G^{\prime}$ (top panel) and $G^{\prime \prime}$ (lower panel) are shown as functions of strain $\gamma$ at a constant frequency of $1 \mathrm{~Hz}$ for different particle concentrations, as labeled. The responses on successively both increasing and decreasing strain are shown. An elastic response could not be detected for the lowest concentration $c=0.2994$.

persions [39]. For the higher concentrations we cannot discern any firm trend in the strain value delineating linear and nonlinear behavior. The exception is perhaps the two highest concentrations, where $G^{\prime \prime}$ shows some strain dependence at lower strains than it does at lower concentrations. However, here the $G^{\prime \prime}$ data are less reliable as the elastic response dominates strongly. Usually one finds that the linear response regime shifts to lower strains with increasing concentration [11,39], but for a limited range of concentrations around the glass transition the extent of this shift may be minor unless one moves further into the glassy state [68].

Monitoring the linear viscoelastic response as a function of frequency, we obtain the elastic modulus, shown in Fig. 6, and the loss modulus, shown in Fig. 7. For concentrations up to $\phi^{H S} \approx 0.45$ the system behaves as a viscous liquid, for which the input work is dissipated mainly by viscous friction. Above this concentration the response crosses over to viscoelastic and becomes progressively more elastic as the concentration is further increased. At the higher concentrations, the elastic modulus becomes independent of frequency and a minimum in the loss modulus is observed for the three highest concentrations.

Since crystalline solid dispersions exhibit both a lowfrequency $G^{\prime}$ plateau and a minimum at intermediate fre-

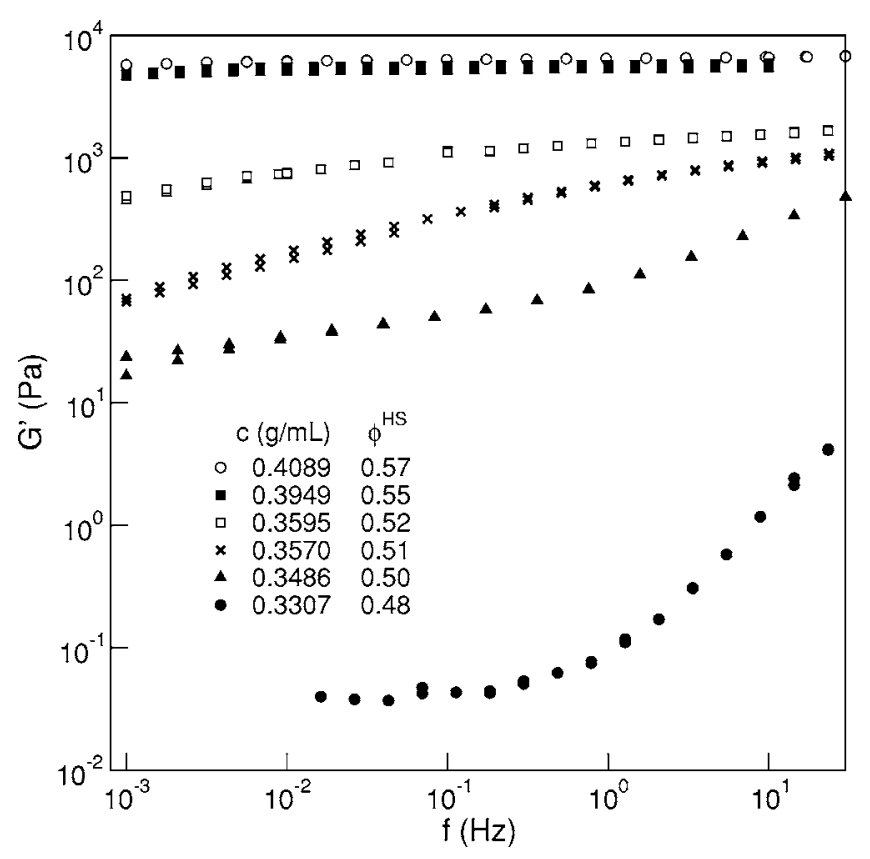

FIG. 6. Storage modulus $G^{\prime}$ as a function of frequency and particle concentration, as labeled. Measurements were conducted in the linear response regime at a strain value of $0.5 \%$. The responses on successively both increasing and decreasing frequency are shown.

quencies in $G^{\prime \prime}$ [69], these are trademarks not necessarily of glassy behavior but general signatures of solidlike behavior. However, as we have shown that our dispersions remain disordered, we can attribute the viscoelastic solidlike response

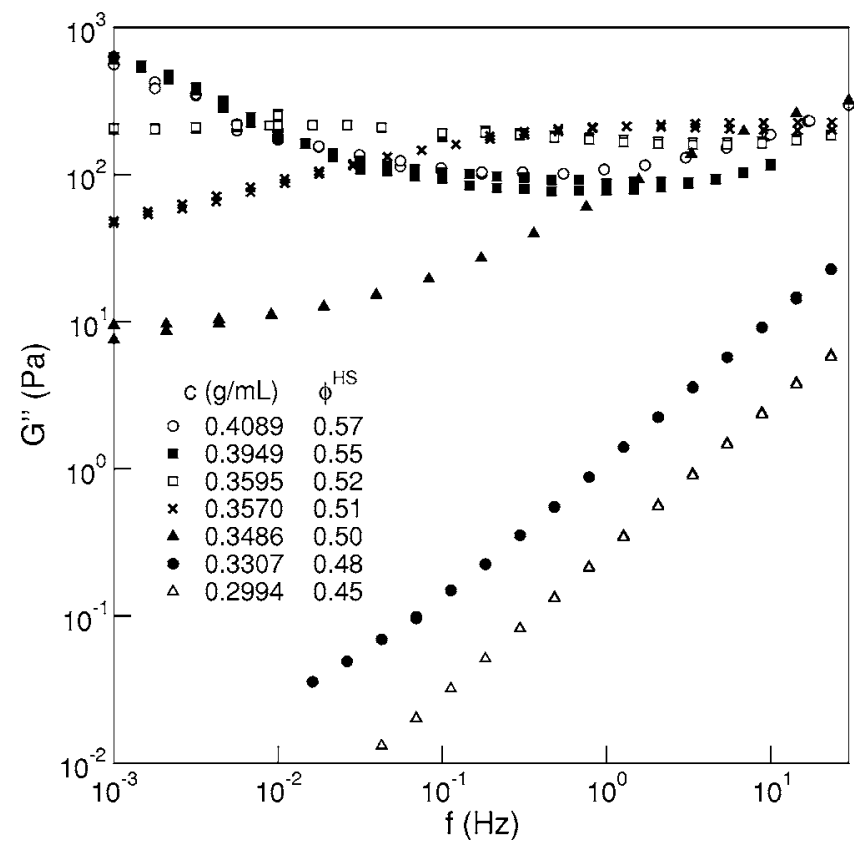

FIG. 7. Loss modulus $G^{\prime \prime}$ as a function of frequency and particle concentration, as labeled. Measurements were conducted within the linear viscoelastic region at a strain value of $0.5 \%$. The responses on successively both increasing and decreasing frequency are shown. 
to glassy behavior in connection with traversing the nonergodicity transition. They are also qualitatively consistent with observations on disordered hard-sphere [11] and polymerically stabilized particle [39-43] dispersions and are predicted by MCT [28]. MCT also predicts a low-frequency maximum in $G^{\prime \prime}$, connected to the slowest-relaxing mode in the diffusive dynamics. Such a maximum appears in our data close to the glass transition, but moves rapidly out of the accessible frequency window; it can be seen more clearly in other measurements on similar systems [39].

The low-frequency plateau in the elastic modulus is obtained for a concentration of $\phi^{H S} \approx 0.55$, very close to where the dynamics becomes nonergodic and where a yield stress in steady-shear measurements can be defined. In addition, for this concentration we find that the peak stress in the oscillatory amplitude sweep at the critical (yield) strain is very close to the yield stress obtained from steady shear measurements, $\sigma_{y} \approx \gamma_{c} G^{\prime}(\omega \rightarrow 0)$, in agreement with previous findings $[70,71]$.

\section{CONCLUSIONS}

The structure, dynamics, and rheology of aqueous dispersions of colloids stabilized by grafted PEG have been studied under good solvent conditions. As the particles are moderately polydisperse, the static structure remains disordered and the "phase diagram" consists simply of a glass transition point. The diffusive dynamics is probed by DLS measurements using 3D cross correlation on dilution series. Similarly to hard-sphere and other dispersions, a two-step decay in the intensity correlation function sets in at sufficiently high particle concentrations. The precise evolution of the slow relaxation with increasing particle concentration appears to differ from hard-sphere behavior. A glass transition is identified as the particle concentration at which the intensity correlation function obtains a nondecaying component, indicative of an arrest of large-scale particle motion and nonergodic dynamics.

Just as relaxations of large-scale concentration fluctuations become slow close to the glass transition, so do relaxations of shear stress fluctuations. This translates into slow relaxation of small-amplitude structural deformations, such that energy is stored in the distorted microstructure during the oscillation cycle. The resulting elastic response becomes progressively more pronounced on approach to the glassy state, leading to a frequency-independent elastic modulus at, or close to, the glass transition concentration. The magnitude of the plateau modulus is closely connected to a yield stress that can be extracted from steady shear measurements close to the same critical particle concentration.

The results of this study confirm the overall qualitative scenario close to the colloidal glass transition; in particular, we find that for the same system the long-time plateau in the intensity correlation function, the yield stress, and the lowfrequency $G^{\prime}$ plateau all appear at or very close to the same particle concentration. Thus, our experiments lends further support to the glass transition dynamics being the key to understanding the low-shear, low-frequency rheology of colloidal dispersions $[8,9,11,22,28,48]$.

\section{ACKNOWLEDGMENTS}

Financial support by the Swedish Foundation for Strategic Research (SSF, Program for Colloid and Interface Technology), the Swedish Research Council, is gratefully acknowledged. J.B. is supported by a grant from the Knut and Alice Wallenberg Foundation. Further support by the State Secretariat for Education and Research (SBF No. 03.0566), the Marie Curie Network on Dynamical Arrest of Soft Matter and Colloids (Grant No. MRTN-CT-2003-504712), and the Swiss National Science Foundation is gratefully acknowledged.
[1] W. B. Russel, D. A. Saville, and W. R. Schowalter, Colloidal Dispersions (Cambridge University Press, New York, 1989).

[2] T. Shikata and D. S. Pearson, J. Rheol. 38, 601 (1994).

[3] S.-E. Phan, W. B. Russel, Z. Chang, J. Zhu, P. M. Chaikin, J. H. Dunsmuir, and R. H. Ottewill, Phys. Rev. E 54, 6633 (1996).

[4] S. P. Meeker, W. C. K. Poon, and P. N. Pusey, Phys. Rev. E 55, 5718 (1997).

[5] J. F. Brady, Chem. Eng. Sci. 56, 2921 (2001).

[6] R. A. Lionberger and W. B. Russel, Adv. Chem. Phys. 111, 399 (2000).

[7] J. Bergenholtz, Curr. Opin. Colloid Interface Sci. 6, 484 (2001).

[8] M. Fuchs and M. E. Cates, Faraday Discuss. 123, 267 (2003).

[9] M. Fuchs and M. E. Cates, Phys. Rev. Lett. 89, 248304 (2002).

[10] B. J. Ackerson, J. Rheol. 34, 553 (1990).

[11] T. G. Mason and D. A. Weitz, Phys. Rev. Lett. 75, 2770 (1995).

[12] L. Marshall and C. F. Zukoski, J. Phys. Chem. 94, 1164
(1990).

[13] P. N. Segre, S. P. Meeker, P. N. Pusey, and W. C. K. Poon, Phys. Rev. Lett. 75, 958 (1995).

[14] P. N. Pusey and W. van Megen, Nature (London) 320, 340 (1986).

[15] P. N. Pusey and W. van Megen, Phys. Rev. Lett. 59, 2083 (1987).

[16] W. van Megen and S. M. Underwood, Phys. Rev. E 47, 248 (1993).

[17] W. van Megen and S. M. Underwood, Phys. Rev. E 49, 4206 (1994).

[18] E. R. Weeks, J. C. Crocker, A. C. Levitt, A. Schofield, and D. A. Weitz, Science 287, 627 (2000).

[19] W. K. Kegel and A. van Blaaderen, Science 287, 290 (2000).

[20] N. B. Simeonova and W. K. Kegel, Phys. Rev. Lett. 93, 035701 (2004).

[21] W. van Megen, T. C. Mortensen, S. R. Williams, and J. Muller, Phys. Rev. E 58, 6073 (1998).

[22] Z. Cheng, J. Zhu, P. M. Chaikin, S.-E. Phan, and W. B. Russel, Phys. Rev. E 65, 041405 (2002). 
[23] W. C. K. Poon, MRS Bull. 29, 96 (2004).

[24] U. Bengtzelius, W. Götze, and A. Sjölander, J. Phys. C 17, 5915 (1984).

[25] W. Götze and L. Sjögren, Rep. Prog. Phys. 55, 241 (1992).

[26] W. Götze and L. Sjögren, Phys. Rev. A 43, 5442 (1991).

[27] W. Götze, J. Phys.: Condens. Matter 11, A1 (1999).

[28] M. Fuchs and M. R. Mayr, Phys. Rev. E 60, 5742 (1999).

[29] A. J. Banchio, G. Nägele, and J. Bergenholtz, J. Chem. Phys. 111, 8721 (1999).

[30] G. Petekidis, D. Vlassopoulos, and P. N. Pusey, J. Phys.: Condens. Matter 16, S3955 (2004).

[31] C. Beck, W. Härtl, and R. Hempelmann, J. Chem. Phys. 111, 8209 (1999).

[32] E. Bartsch, V. Frenz, J. Baschnagel, W. Schärtl, and H. Silescu, J. Chem. Phys. 106, 3743 (1997).

[33] B. J. Maranzano and N. J. Wagner, Rheol. Acta 39, 483 (2000).

[34] D. H. Napper, Polymeric Stabilization of Colloidal Dispersions (Academic Press, London, 1983).

[35] G. Petekidis, J. Gapinski, P. Seymour, J. S. van Duijneveldt, D. Vlassopoulos, and G. Fytas, Phys. Rev. E 69, 042401 (2004).

[36] I. Deike, M. Ballauff, N. Willenbacher, and A. Weiss, J. Rheol. 45, 709 (2001).

[37] W. J. Frith, P. d'Haene, R. Buscall, and J. Mewis, J. Rheol. 40, 531 (1996).

[38] J. Mewis and G. Biebaut, J. Rheol. 45, 799 (2001).

[39] W. J. Frith, T. A. Strivens, and J. Mewis, J. Colloid Interface Sci. 139, 55 (1990).

[40] H. J. Ploehn and J. W. Goodwin, in Polymers as Rheology Modifiers, edited by D. N. Schulz and J. E. Glass, ACS Symposium Series No. 462 (American Chemical Society, Washington, DC, 1991).

[41] H. Senff, W. Richtering, C. Norhausen, A. Weiss, and M. Ballauff, Langmuir 15, 102 (1999).

[42] P. A. Nommensen, M. H. G. Duits, D. van den Ende, and J. Mellema, Langmuir 16, 1902 (2000).

[43] P. A. Nommensen, D. van den Ende, M. H. G. Duits, and J. Mellema, Langmuir 17, 5757 (2001).

[44] C. Prestidge and T. F. Tadros, J. Colloid Interface Sci. 124, 660 (1988).

[45] J. Mewis, W. J. Frith, T. A. Strivens, and W. B. Russel, AIChE J. 35, 415 (1989).

[46] W. Liang, T. F. Tadros, and P. F. Luckham, J. Colloid Interface Sci. 153, 131 (1992).

[47] P. A. Nommensen, M. H. G. Duits, D. van den Ende, and J.
Mellema, Phys. Rev. E 59, 3147 (1999)

[48] M. Fuchs and M. Ballauff, J. Chem. Phys. 122, 094707 (2005).

[49] M. Zackrisson, A. Stradner, P. Schurtenberger, and J. Bergenholtz, Langmuir 21, 10835 (2005).

[50] A. Brindley, S. S. Davis, M. C. Davies, and J. F. Watts, J. Colloid Interface Sci. 171, 150 (1995)

[51] E. Overbeck, C. Sinn, T. Palberg, and K. Schätzel, Colloids Surf., A 122, 83 (1997).

[52] C. Urban and O. Schurtenberger, J. Colloid Interface Sci. 207, 150 (1998).

[53] J.-Z. Xue, D. J. Pine, S. T. Milner, X.-1. Wu, and P. M. Chaikin, Phys. Rev. A 46, 6550 (1992).

[54] J. Lauger, K. Wollny, and S. Huck, Rheol. Acta 41, 356 (2002).

[55] F. E. Bailey and J. V. Koleske, Poly(Ethylene Oxide) (Academic Press, New York, 1976).

[56] G. Dietler and D. S. Cannell, Phys. Rev. Lett. 60, 1852 (1988).

[57] J. P. Wilcoxon, J. Phys. Chem. 94, 7588 (1990).

[58] C. van den Broeck, F. Lostak, and H. N. W. Lekkerkerker, J. Chem. Phys. 74, 2006 (1981).

[59] J. L. Lebowitz, Phys. Rev. 133, 895 (1964).

[60] L. Blum and G. Stell, J. Chem. Phys. 71, 42 (1979).

[61] W. L. Griffith, R. Triolo, and A. L. Compere, Phys. Rev. A 35, 2200 (1987).

[62] B. D'Aguanno and R. Klein, Phys. Rev. A 46, 7652 (1992).

[63] P. Szymczak and B. Cichocki, J. Chem. Phys. 121, 3329 (2004).

[64] P. N. Pusey, in Liquids, Freezing and Glass Transition., edited by J.-P. Hansen, D. Levesque, and J. Zinn-Justin (NorthHolland, Amsterdam, 1991).

[65] C. G. de Kruif, E. M. F. van Iersel, A. Vrij, and W. B. Russel, J. Chem. Phys. 83, 4717 (1985).

[66] D. A. R. Jones, B. Leary, and D. V. Boger, J. Colloid Interface Sci. 147, 479 (1991).

[67] W. J. Frith, J. Mewis, and T. A. Strivens, Powder Technol. 51, 27 (1987).

[68] G. Petekidis, A. Moussaid, and P. N. Pusey, Phys. Rev. E 66, 051402 (2002).

[69] B. van der Vorst, D. van den Ende, and J. Mellema, J. Rheol. 39, 1183 (1995).

[70] T. G. Mason, J. Bibette, and D. A. Weitz, J. Colloid Interface Sci. 179, 439 (1996).

[71] W. B. Russel and M. C. Grant, Colloids Surf., A 161, 271 (2000). 